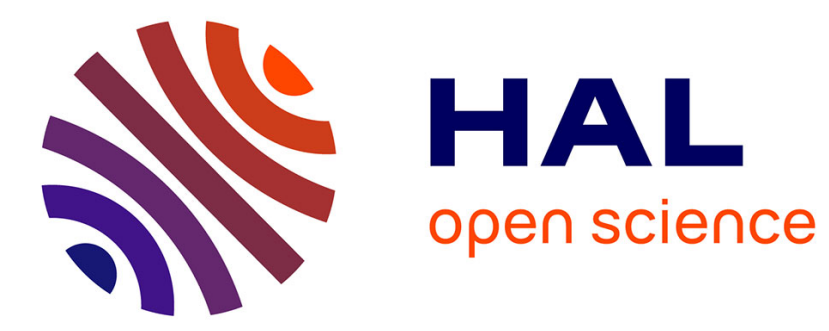

\title{
Convergence Analysis of an online Approach to Parameter Estimation Problems Based on Binary Observations
}

Kian Jafaridinani, Jérome Juillard, Morgan Roger

\section{- To cite this version:}

Kian Jafaridinani, Jérome Juillard, Morgan Roger. Convergence Analysis of an online Approach to Parameter Estimation Problems Based on Binary Observations. Automatica, 2012, 48 (11), pp.28372842. 10.1016/j.automatica.2012.05.050 . hal-00747809

\section{HAL Id: hal-00747809 \\ https://hal-centralesupelec.archives-ouvertes.fr/hal-00747809}

Submitted on 2 Nov 2012

HAL is a multi-disciplinary open access archive for the deposit and dissemination of scientific research documents, whether they are published or not. The documents may come from teaching and research institutions in France or abroad, or from public or private research centers.
L'archive ouverte pluridisciplinaire HAL, est destinée au dépôt et à la diffusion de documents scientifiques de niveau recherche, publiés ou non, émanant des établissements d'enseignement et de recherche français ou étrangers, des laboratoires publics ou privés. 


\title{
Convergence analysis of an online approach to parameter estimation problems based on binary observations *
}

\author{
Kian Jafari, Jérôme Juillard, Morgan Roger \\ SUPELEC - Department of Signal Processing and Electronic Systems, Gif-sur-Yvette, France
}

\begin{abstract}
In this paper, we present an online identification method to the problem of parameter estimation from binary observations. A recursive identification algorithm with low-storage requirements and computational complexity is derived. We prove the convergence of this method provided that the input signal satisfies a strong mixing property. Some simulation results are then given in order to illustrate the properties of this method under various scenarios. This method is appealing in the context of micro-electronic devices since it only requires a 1-bit analog-to-digital converter, with low power consumption and minimal silicon area.
\end{abstract}

Key words: System identification; parameter estimation; binary signals; LMS algorithm; convergence analysis.

\section{Introduction}

The parameter estimation problem for systems with binary (or quantized) outputs is one of the absorbing questions in a wide range of applications (Wang et al., 2003; Juillard et al., 2009). Networked control systems, sensor networks and telecommunications are some examples in this domain (Zhao et al., 2007). The use of such methods is mostly motivated by the fact that low-resolution observations are much cheaper or easier to obtain than high-resolution ones.

The method presented in this paper is aimed at the test of micro-electronic devices, such as MEMS (Microelectromechanical systems). It is known that as characteristic dimensions become smaller, the dispersions afflicting electronic devices tend to become larger. Variations in the fabrication process (Deb, 2005) or changes in the operating conditions, such as temperature, pressure or humidity are typical sources of dispersion (Colinet \& Juillard, 2010). It is then desirable to integrate self-test (and self-tuning) features, such as parameter estimation routines, in each device in order to compensate the fabrication dispersions and adapt to changing conditions. Ideally, this self-test feature should be as lowcost as possible, especially in terms of silicon area. Thus,

\footnotetext{
* This paper was not presented at any IFAC meeting. Corresponding author K. Jafari and J. Juillard. Tel. +33169 8514 24. Fax +33169851429.

Email addresses: Kian. Jafari@ieee.org (Kian Jafari), Jerome. Juillard@supelec.fr (Jérôme Juillard), Morgan.Roger@supelec.fr (Morgan Roger).
}

parameter estimation routines based on binary observations are very appealing because they only require the integration of a 1-bit analog-to-digital converter (ADC) (Van de Plassche, 2005).

Many significant results have been established for system identification based on binary (or quantized) data in the last few years (Rafajlowicz, 1996; Wigren, 1997, 1998; Wang et al., 2003, 2006; Bai \& Reyland Jr., 2008; Gustafsson \& Karlsson, 2009; Colinet \& Juillard, 2010). A brief summary of these works can be found in (Jafari et al., 2010), where an approach based on the least-mean squares (LMS) algorithm was introduced.

In particular, Wigren has developed an approach to the system identification problem based on quantized observations provided that there is signal energy around the switch points. The global convergence is proved for a finite impulse response (FIR) system with an arbitrary and known output quantizer under the assumption that the quantizer has at least one threshold value different from zero, building on arguments from (Ljung, 1999). Wang and his co-authors have also presented a method for estimating parameters from binary (or quantized) data. The unknown system is excited by a periodic signal and the threshold of the quantizer is randomly specified by a partially known dithering signal. It is proved that the cumulative distribution function of the threshold does not have to be known a priori and it can be estimated along with the system parameters.

The present work differs from Wigren's method in the sense that it does not rely on a pseudo-gradient of a leastsquares criterion and it does not require the threshold 


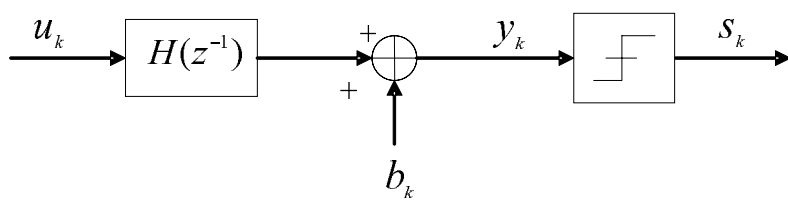

Fig. 1. Block diagram of the system model. Signal $y_{k}$ is not available for measurement.

value to be different from zero. Furthermore, it does not require a varying threshold, as opposed to Wang's approach.

In this paper, we introduce a new recursive identification method based on binary observations. This approach is an online method which can rule out the existing techniques relying on batch-data-based methods, e.g. (Colinet \& Juillard, 2010), or on maximum likelihood algorithms in terms of storage requirements and computational complexity. The convergence of the algorithm, which relies on an adaptive regulative coefficient, is proved (as opposed to (Jafari et al., 2010)). Furthermore, the influence of the relaxation coefficient, of the initial point and of the quantization of the input are investigated by simulation.

The structure of the article is the following. In section 2 , the notations and framework are given. In section 3, the algorithm for estimating the unknown system parameters from binary outputs is derived. The asymptotic convergence of the proposed algorithm is demonstrated under some mild assumptions. Section 4 illustrates the behaviour of the algorithm when these assumptions are partially relaxed. Conclusions and perspectives are drawn in section 5 .

\section{Notations and frameworks}

The known input signal $u_{k}$ is filtered by a discrete-time invariant linear system $H\left(z^{-1}\right)$ to produce the system output $y_{k}$ at time $k$ (Fig. 1).

$H$ has an impulse response of length $L$, i.e. it can be represented by a column vector $\boldsymbol{\theta}=\left(\theta_{l}\right)_{l=1}^{L}$. Let $\hat{\boldsymbol{\theta}}_{k}$ be the estimated vector of parameters (of length $L$ ) at time $k$ and $\hat{\boldsymbol{\theta}}_{1}$ be the initial estimate. Let also, $b_{k}$ be an additive noise at the system output. The output and the estimated output can be expressed as:

$$
\left\{\begin{array}{l}
y_{k}=\boldsymbol{\theta}^{\top} \boldsymbol{\phi}_{k}+b_{k} \\
\hat{y}_{k}=\hat{\boldsymbol{\theta}}_{k}^{\top} \boldsymbol{\phi}_{k}
\end{array},\right.
$$

where $\phi_{k}=\left[u_{k}, u_{k-1}, \ldots, u_{k-L+1}\right]^{\top}$ is the vector of inputs at time $k$. The system output then goes through a 1-bit ADC so that only the sign $s_{k}=S\left(y_{k}\right)$ of the system output is known, where

$$
\begin{cases}s_{k}=1, & \text { if } y_{k} \geq 0 \\ s_{k}=-1, & \text { otherwise }\end{cases}
$$

Our goal is to develop a recursive estimation method to estimate $\boldsymbol{\theta}$ from $\hat{\boldsymbol{\theta}}_{k}$ based on $N$ observations of $u_{k}, s_{k}$. Because of the absence of dither signal at the comparator input, it is only possible to identify $\boldsymbol{\theta}$ up to a positive multiplicative constant (Colinet \& Juillard, 2010). Without loss of generality, we then consider $\|\boldsymbol{\theta}\|=1$ in the remaining of the paper. In summary, we are going to estimate the coefficients $\boldsymbol{\theta}$ of a FIR filter (of length $L$ ) such that $\|\boldsymbol{\theta}\|=1$ using

- the binary observations $s_{k}$ of the sign of the filter output.

- the inputs $u_{k}$ of the filter (not necessarily binary).

We assume the following prior information on the system.

Assumption $1 v_{1}=\hat{\boldsymbol{\theta}}_{1}^{\top} \boldsymbol{\theta} \geq 0$.

Remark 1 It is rather simple to verify assumption 1. For example, a positive constant signal is used as input. If the sign of the output is positive, it means that the sign $S_{g}$ of the static gain is positive $\left(S_{g}=1\right)$, otherwise $S_{g}=-1$. Choosing

$$
\hat{\boldsymbol{\theta}}_{1}=\frac{S_{g}}{L} \times[1,1, \ldots, 1]^{\top}
$$

then ensures that assumption 1 is verified.

Assumption $2 \phi_{k}$ is a random process such that

- the probability density function (pdf) of $\frac{\phi_{k}}{\left\|\phi_{k}\right\|}$ is nonzero on the unit sphere.

- $\phi_{k}$ verifies the $\alpha$-mixing condition (Rosenblatt, 1956).

Remark 2 The first condition in assumption 2 ensures that $\phi_{k}$ may be in any angular sector of $\mathbb{R}^{L}$ and the second condition guarantees that $\phi_{k}$ and $\phi_{k+l}$ can be considered as independent for large enough l. An input signal verifying assumption 2 is a sequence of i.i.d. random variables with a continuous pdf whose support contains an interval of the form $[-a, a], a>0$ (e.g. uniform and centered) (Bai $\&$ Reyland Jr., 2008).

In the following section, our approach is presented and we study the convergence of sequences $\hat{\boldsymbol{\theta}}_{k}$ to $\boldsymbol{\theta}$ based on an LMS treatment of the offline methods proposed in (Colinet \& Juillard, 2010; Juillard et al., 2009). The proof of the convergence is first established in the noisefree case:

Assumption $3 b_{k}=0$,

then the convergence of the algorithm in the more realistic case when $b_{k} \neq 0$ is shown by Monte Carlo simulations. 


\section{Identification algorithm and its convergence}

\subsection{Basic approach to the problem}

In classical offline identification methods (i.e. when the output of the system is not quantized (Walter \& Pronzato, 1997; Ljung, 1999)), the quadratic error is defined as $\sum_{k}\left(y_{k}-\hat{y}_{k}\right)^{2}$. Thus, the instantaneous estimation error is $\tilde{E}_{k}=y_{k}-\hat{y}_{k}$. As a consequence, the following algorithm is used in the classical LMS approach (Haykin, 2001):

$$
\hat{\boldsymbol{\theta}}_{k+1}=\hat{\boldsymbol{\theta}}_{k}-\alpha_{k} \frac{\partial \tilde{E}_{k}^{2}}{\partial \hat{\theta}}
$$

where $\alpha_{k}>0$ must verify some conditions to guarantee the stability and convergence of the algorithm.

In (Colinet \& Juillard, 2010; Juillard et al., 2009), it is shown that the offline identification of $\boldsymbol{\theta}$ based on binary observations can be achieved by minimizing a quadratic error of the form

$$
E^{2}=\frac{1}{4} \sum_{k=1}^{N}\left(s_{k}-\hat{s}_{k}\right)^{2} \hat{y}_{k}^{2}
$$

where $N$ is the number of observations. We propose to extend this offline method to an online approach by using (4) and the following definition of the instantaneous error:

$$
\tilde{E}_{k}=\frac{1}{2}\left|s_{k}-\hat{s}_{k}\right| \hat{y}_{k}
$$

Using (1), (6) and the fact that $\left|s_{k}-\hat{s}_{k}\right|^{2}=2\left|s_{k}-\hat{s}_{k}\right|$, (4) can be developed as

$$
\hat{\boldsymbol{\theta}}_{k+1}=\hat{\boldsymbol{\theta}}_{k}-\alpha_{k}\left|s_{k}-\hat{s}_{k}\right| \hat{y}_{k} \boldsymbol{\phi}_{k}
$$

Equation (7) above is equivalent to

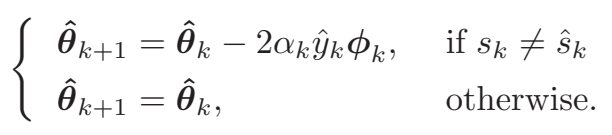

Since it is impossible to estimate the static gain of $\boldsymbol{\theta}$ in the absence of dither, we shall choose $\alpha_{k}$ so that $\left\|\hat{\boldsymbol{\theta}}_{k}\right\|$ remains constant. To this end, we use

$$
\alpha_{k}=\frac{1}{\phi_{k}^{\top} \phi_{k}}
$$

for which it is possible to verify that $\left\|\hat{\boldsymbol{\theta}}_{k+1}\right\|=\left\|\hat{\boldsymbol{\theta}}_{k}\right\|$. Without loss of generality, we impose $\left\|\hat{\boldsymbol{\theta}}_{k}\right\|=1$. Let us consider the sequence $v_{k}=\hat{\boldsymbol{\theta}}_{k}^{\top} \boldsymbol{\theta}$. Projecting (7) on $\boldsymbol{\theta}$ under assumption 3 , we obtain:

$$
v_{k+1}=v_{k}-\alpha_{k}\left|s_{k}-\hat{s}_{k}\right| \hat{y}_{k} y_{k}
$$

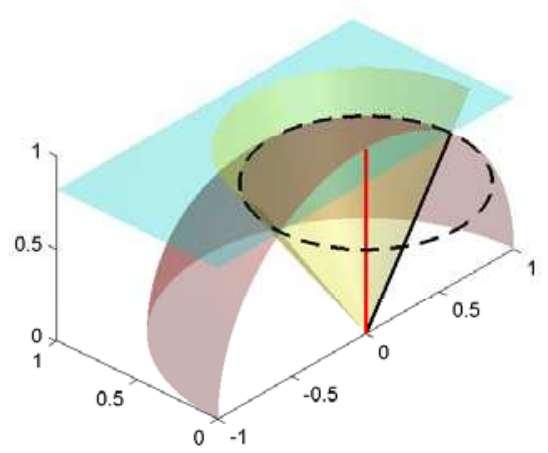

Fig. 2. As $k$ goes to infinity, $\hat{\boldsymbol{\theta}}_{k}$ revolves around $\boldsymbol{\theta}$ on a circle orthogonal to $\boldsymbol{\theta}$, defined as the intersection of the unit sphere and a cone of revolution with axis $\boldsymbol{\theta}$ and half-angle $\cos ^{-1}\left(v_{\infty}\right)$.

The product $\hat{y}_{k} y_{k}$ being negative if $s_{k} \neq \hat{s}_{k}$, this sequence is monotonically increasing. Furthermore, the Cauchy-Schwartz inequality

$$
\hat{\boldsymbol{\theta}}_{k}^{\top} \boldsymbol{\theta} \leq\left\|\hat{\boldsymbol{\theta}}_{k}\right\|\|\boldsymbol{\theta}\|=\|\boldsymbol{\theta}\|
$$

implies that the sequence $\left(v_{k}\right)$ is bounded from above and thus, converges to a limit $v_{\infty}$. This means that:

- either $\hat{\boldsymbol{\theta}}_{k}$ converges to a limit $\hat{\boldsymbol{\theta}}_{\infty}$.

- or $\hat{\boldsymbol{\theta}}_{k}$ is drawn towards a cone whose axis is oriented by $\boldsymbol{\theta}$ with half-angle $\cos ^{-1}\left(v_{\infty}\right)$ (Fig. 2) without converging, for example, orbiting around $\theta$ on and on.

Although simulations seem to show that the second case is never met in practice, for a wide class of inputs, we have not been able to prove the convergence of (7) without resorting to overly strong hypotheses ${ }^{1}$. However, one may construct another sequence of $\hat{\boldsymbol{\theta}}_{k}$ in order to avoid getting stuck on a cone. Intuitively, this sequence may be constructed from a combination of $\hat{\boldsymbol{\theta}}_{k}$ and of the $\hat{\boldsymbol{\theta}}_{k+1}$ derived from (7). If $k$ is large enough so that $\hat{\boldsymbol{\theta}}_{k}$ and $\hat{\boldsymbol{\theta}}_{k+1}$ are close to the limit cone, the angle between $\boldsymbol{\theta}$ and the vector resulting from any convex combination of $\hat{\boldsymbol{\theta}}_{k}$ and $\hat{\boldsymbol{\theta}}_{k+1}$ will necessarily be smaller than $\cos ^{-1}\left(v_{\infty}\right)$. Thus, it should be possible to relax (7) to ensure convergence. This is studied in the next subsection.

\subsection{Relaxed approach}

The following relaxed algorithm is considered:

$$
\hat{\boldsymbol{\theta}}_{k+1}=\frac{\hat{\boldsymbol{\theta}}_{k}-\mu \alpha_{k}\left|s_{k}-\hat{s}_{k}\right| \hat{y}_{k} \boldsymbol{\phi}_{k}}{w_{k}}
$$

1 Note that, for example, the convergence of (7) can be proved by assuming that the data is absolutely linearly separable (i.e. $\exists \eta>0|| y_{k} \mid>\eta, \forall k$ ) and the training set is repeated over and over (Haykin, 2001). However, these hypotheses do not hold in the context of this paper. 
where $0<\mu<1$ and

$$
w_{k}=\sqrt{1-2 \mu(1-\mu) \alpha_{k}\left|s_{k}-\hat{s}_{k}\right| \hat{y}_{k}^{2}} \leqslant 1
$$

is a normalizing factor so that $\left\|\hat{\boldsymbol{\theta}}_{k}\right\|=1, \forall k$.

Remark 3 If $\mu=1$, algorithm (7) is obtained. On the other hand, if $\mu=0$, we get $\hat{\boldsymbol{\theta}}_{k}=\hat{\boldsymbol{\theta}}_{1}, \forall k$.

Theorem 4 Under assumptions 1, 2 and 3, the sequence defined by (12)-(13) and (9) converges surely to $\boldsymbol{\theta}, \forall \mu \in$ $(0,1)$.

PROOF. When $0<\mu<1$, the $\hat{\boldsymbol{\theta}}_{k+1}$ defined by (12) is a convex combination of $\hat{\boldsymbol{\theta}}_{k}$ (with weight $1-\mu$ ) and of the $\hat{\boldsymbol{\theta}}_{k+1}$ defined by (7) (with weight $\mu$ ). Projecting (12) on $\boldsymbol{\theta}$, we get

$$
v_{k+1}=\frac{v_{k}-\mu \alpha_{k}\left|s_{k}-\hat{s}_{k}\right| \hat{y}_{k} y_{k}}{w_{k}} .
$$

Since $\mu \alpha_{k}\left|s_{k}-\hat{s}_{k}\right| \hat{y}_{k} y_{k} \leq 0,0 \leq w_{k} \leq 1$ and $v_{1} \geq 0$, the sequence $\left(v_{k}\right)$ is monotonically increasing. As it is also bounded from above (11), it converges and then $\frac{v_{k}}{v_{k+1}} \stackrel{k \rightarrow \infty}{\longrightarrow} 1$.

From (14) and (13), $\frac{v_{k}}{v_{k+1}} \leqslant w_{k} \leqslant 1$, hence $w_{k} \stackrel{k \rightarrow \infty}{\longrightarrow} 1$. Considering the definition of $w_{k}$ (13), this proves that $\alpha_{k}\left|s_{k}-\hat{s}_{k}\right| \hat{y}_{k}^{2} \stackrel{k \rightarrow \infty}{\longrightarrow} 0$. Furthermore, projecting (12) on $\hat{\boldsymbol{\theta}}_{k}$ yields:

$$
\hat{\boldsymbol{\theta}}_{k+1}^{\top} \hat{\boldsymbol{\theta}}_{k}=\frac{1-\mu \alpha_{k}\left|s_{k}-\hat{s}_{k}\right| \hat{y}_{k}^{2}}{w_{k}} .
$$

Hence, $\hat{\boldsymbol{\theta}}_{k+1}^{\top} \hat{\boldsymbol{\theta}}_{k} \stackrel{k \rightarrow \infty}{\longrightarrow} 1$. On the other hand, projecting (12) on $\hat{\boldsymbol{\theta}}_{k+1}$ yields:

$$
1=\frac{\hat{\boldsymbol{\theta}}_{k}^{\top} \hat{\boldsymbol{\theta}}_{k+1}-\mu \alpha_{k}\left|s_{k}-\hat{s}_{k}\right| \hat{y}_{k} \boldsymbol{\phi}_{k}^{\top} \hat{\boldsymbol{\theta}}_{k+1}}{w_{k}}
$$

which implies $\alpha_{k}\left|s_{k}-\hat{s}_{k}\right| \hat{y}_{k} \boldsymbol{\phi}_{k}^{\top} \hat{\boldsymbol{\theta}}_{k+1} \stackrel{k \rightarrow \infty}{\longrightarrow} 0$, since $w_{k}$ and $\hat{\boldsymbol{\theta}}_{k}^{\top} \hat{\boldsymbol{\theta}}_{k+1}$ converge to 1 as seen above. Furthermore, one proves that $\alpha_{k+1}\left|s_{k+1}-\hat{s}_{k+1}\right| \hat{y}_{k+1} \boldsymbol{\phi}_{k+1}^{\top} \hat{\boldsymbol{\theta}}_{k} \stackrel{k \rightarrow \infty}{\longrightarrow} 0$ by projecting (12) on $\alpha_{k+1}\left|s_{k+1}-\hat{s}_{k+1}\right| \hat{y}_{k+1} \phi_{k+1}^{\top}$ and using the Cauchy-Schwartz inequality on the second term of the right-hand side. Replacing $k$ by $k+1$ in (12) and projecting the resulting equation on $\hat{\theta}_{k}$, we obtain an expression of $\hat{\boldsymbol{\theta}}_{k+2}^{\top} \hat{\boldsymbol{\theta}}_{k}$ as a function of quantities whose convergence is already established. It is then straightforward that $\hat{\boldsymbol{\theta}}_{k+2}^{\top} \hat{\boldsymbol{\theta}}_{k} \stackrel{k \rightarrow \infty}{\longrightarrow} 1$ and by induction,

$$
\forall l, \quad \hat{\boldsymbol{\theta}}_{k+l}^{\top} \hat{\boldsymbol{\theta}}_{k} \stackrel{k \rightarrow \infty}{\longrightarrow} 1 .
$$
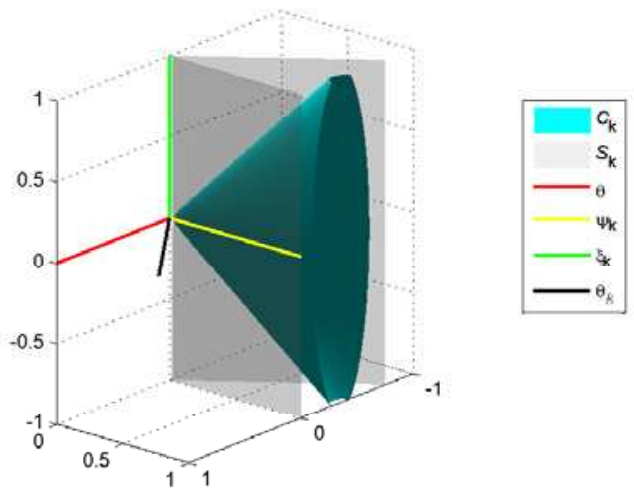

Fig. 3. If $\phi_{k}$ belongs to the sector $S_{k}$, then $s_{k} \neq \hat{s}_{k}$. When $\phi_{k}$ also belongs to the elliptical cone $C_{k}^{1 / 2} \subset S_{k}$, then $s_{k} \neq \hat{s}_{k}$ and $-4 \alpha_{k} \hat{y}_{k} y_{k}>1-v_{k}$.

Consequently, since

$$
\left\|\hat{\boldsymbol{\theta}}_{k+l}-\hat{\boldsymbol{\theta}}_{k}\right\|^{2}=2\left(1-\hat{\boldsymbol{\theta}}_{k+l}^{\top} \hat{\boldsymbol{\theta}}_{k}\right),
$$

the sequence $\hat{\boldsymbol{\theta}}_{k}$ is Cauchy. $\mathbb{R}^{L}$ being complete, $\hat{\boldsymbol{\theta}}_{k}$ converges to a limit $\hat{\boldsymbol{\theta}}_{\infty}$. Let us then show that, provided certain hypotheses hold, $v_{\infty}=\hat{\boldsymbol{\theta}}_{\infty}^{\top} \boldsymbol{\theta}=1$.

As $v_{k}$ is necessarily smaller than 1 , we try to determine under what circumstances $v_{k}$ can be increased by $\epsilon\left(1-v_{k}\right), \epsilon \in(0,1]$. The coefficient $w_{k}$ being smaller than 1 , a sufficient condition for $v_{k+1}-v_{k} \geq \epsilon\left(1-v_{k}\right)$ is that the right-hand term of the numerator of (14) should be greater than $\epsilon\left(1-v_{k}\right)$ when $s_{k} \neq \hat{s}_{k}$, i.e.

$$
2 \mu \alpha_{k} \hat{y}_{k} y_{k}+\epsilon\left(1-v_{k}\right)<0 \text {. }
$$

Multiplying (19) by $\phi_{k}^{\top} \phi_{k}=\alpha_{k}^{-1}$ yields

$$
\boldsymbol{\phi}_{k}^{\top}\left(\mu\left(\boldsymbol{\theta} \hat{\boldsymbol{\theta}}_{k}^{\top}+\hat{\boldsymbol{\theta}}_{k} \boldsymbol{\theta}^{\top}\right)+\epsilon\left(1-v_{k}\right) \boldsymbol{I}_{L}\right) \boldsymbol{\phi}_{k}<0,
$$

where $\boldsymbol{I}_{L}$ is the $L \times L$ identity matrix. Let us decompose $\hat{\boldsymbol{\theta}}_{k}$ as $\hat{\boldsymbol{\theta}}_{k}=v_{k} \boldsymbol{\theta}+\sqrt{1-v_{k}^{2}} \boldsymbol{\psi}_{k}$, where $\boldsymbol{\psi}_{k}$ is a unit vector orthogonal to $\boldsymbol{\theta}$, and $\boldsymbol{\phi}_{k}$ as $\boldsymbol{\phi}_{k}=a_{k} \boldsymbol{\theta}+b_{k} \boldsymbol{\psi}_{k}+c_{k} \boldsymbol{\xi}_{k}$, where $\boldsymbol{\xi}_{k}$ is a unit vector orthogonal to $\boldsymbol{\theta}$ and $\boldsymbol{\psi}_{k}$ (Fig. $3)$. Then, the condition for $s_{k} \neq \hat{s}_{k}$ is derived as:

$$
a_{k}\left(a_{k} v_{k}+b_{k} \sqrt{1-v_{k}^{2}}\right)<0
$$

and (20) can be rewritten as:

$$
\left(\begin{array}{lll}
a_{k} & b_{k} & c_{k}
\end{array}\right) \mathbf{A}_{k}\left(\begin{array}{l}
a_{k} \\
b_{k} \\
c_{k}
\end{array}\right)<0
$$


where

$$
\mathbf{A}_{k}=\left(\begin{array}{ccc}
\epsilon\left(1-v_{k}\right)+2 \mu v_{k} & \mu \sqrt{1-v_{k}^{2}} & 0 \\
\mu \sqrt{1-v_{k}^{2}} & \epsilon\left(1-v_{k}\right) & 0 \\
0 & 0 & \epsilon\left(1-v_{k}\right)
\end{array}\right)
$$

Condition (21) imposes that $\phi_{k}$ should belong to a certain angular sector $S_{k}$ to verify $s_{k} \neq \hat{s}_{k}$ (Fig. 3). The condition (22) cannot be verified unless $\epsilon<\mu$. If such is the case, it imposes that $\phi_{k}$ should belong to the inside of an elliptical cone ${ }^{2}$ contained in $S_{k}$. For example, the elliptical cone $C_{k}^{1 / 2}$ corresponding to $\epsilon=\mu / 2$ is represented in Fig. 3. Its major half-angle is $\alpha=\frac{\pi}{4}$ and its minor half-angle is

$$
\beta_{k}=\tan ^{-1}\left(\sqrt{\frac{1-v_{k}}{3+v_{k}}}\right) .
$$

As $k$ increases, $C_{k}^{1 / 2}$ shrinks (because $\beta_{k}$ is a decreasing function of $v_{k}$ ) while revolving around $\boldsymbol{\theta}$. As $\hat{\boldsymbol{\theta}}_{k}$ is drawn to $\hat{\boldsymbol{\theta}}_{\infty}, C_{k}^{1 / 2}$ goes to a limiting cone $C_{\infty}^{1 / 2}$ with major half-angle $\alpha$ and minor half-angle $\beta_{\infty}$.

Let us suppose $v_{\infty}<1$. Since $v_{k}$ converges, $\forall \eta>0$ there exists $K_{0}$ large enough so that $\forall k>K_{0}, v_{\infty}-v_{k}<\eta$. For example, we take $\eta=\frac{\mu}{4}\left(1-v_{\infty}\right)$. Since $v_{\infty}<1$, the solid angle $\beta_{\infty}$ defined by the limiting cone $C_{\infty}^{1 / 2}$ is greater than 0 . Moreover, it is clear that $\exists K_{1}>K_{0}$ such that

$$
\bigcap_{k>K_{1}} C_{k}^{1 / 2}=C \neq \emptyset .
$$

Consequently, for $k>K_{1}$, any $\phi_{k}$ inside $C$ would increase $v_{k}$ by at least $\frac{\mu}{2}\left(1-v_{\infty}\right)=2 \eta$, which would contradict our initial hypothesis that $v_{\infty}-v_{k}<\eta$.

A necessary condition for $v_{\infty}<1$ is then that

$$
\forall k>K_{1}, P\left(\phi_{k} \in C\right)=0 .
$$

However, $\phi_{k}$ verifies assumption 2 . This means that the probability that a given vector of inputs enters $C$ in finite time after $K_{1}$ is 1 . Thus (25) cannot hold and $v_{\infty}=1$ (i.e. $\hat{\boldsymbol{\theta}}_{\infty}=\boldsymbol{\theta}$ ). This completes the proof of the theorem.

\section{$4 \quad$ Numerical results}

In this section, we show by simulations that, in order to obtain an appropriate performance of the proposed method (called LIMBO for LMS-based Identification Method using Binary Observations) in real applications, assumptions 1, 2 and 3 used in the proof of the convergence do not need to be stringently verified. The influence of the relaxation coefficient $\mu$ on the convergence

\footnotetext{
${ }^{2}$ If $L=2$, the $\phi_{k}$ verifying (19) are inside a two-dimensional angular sector contained in $S_{k}$. The rest of the proof is unaffected.
}

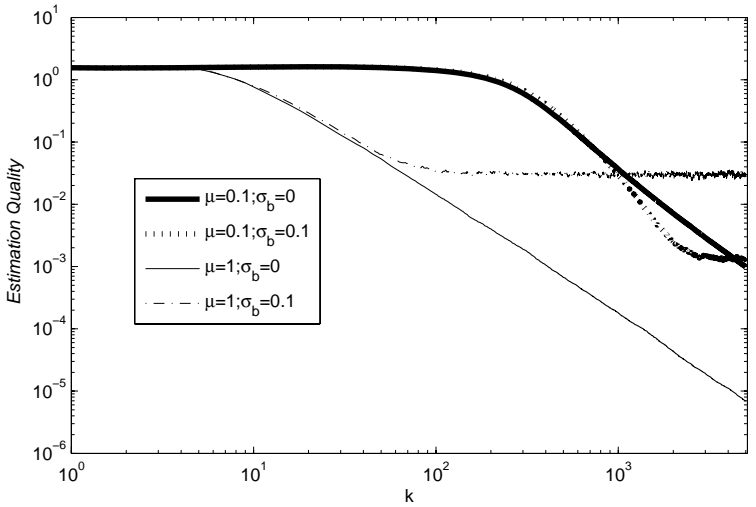

Fig. 4. $80^{t h}$ percentile of $\left(1-v_{k}\right)$ for various values of $\mu$ and $\sigma_{b}$ using a uniformly distributed input signal.

rate is also investigated. We choose the same test case as in (Wigren, 1998): $\boldsymbol{\theta}=\left[\begin{array}{llll}1 & -0.7 & 4 & -2.8\end{array}\right]$. Note that $\boldsymbol{\theta}$ is normalized before starting the LIMBO algorithm. The results of the Monte Carlo simulations presented in this section are based on 5000 realizations of the input signal (and of the measurement noise when $b_{k} \neq 0$ ).

First, we study the influence of $\mu$ and measurement noise. The signal $u_{k}$ is uniformly distributed on $[-1,1]$, $b_{k}$ has a Gaussian distribution with zero mean and variance $\sigma_{b}^{2}$ and both signals are white. The algorithm is initialized with $\hat{\boldsymbol{\theta}}_{1}=\left[\begin{array}{llll}0 & 0 & 0 & 1\end{array}\right]$. Figure 4 illustrates the estimation quality, measured by the $80^{\text {th }}$ percentile of $\left(1-v_{k}\right)$ from the Monte Carlo realizations, for two values of $\mu$ and $\sigma_{b}$. In the noise-free case $\left(\sigma_{b}=0\right)$, the larger $\mu$ is, the faster the convergence of the algorithm is. Moreover, the convergence is obtained even in the non-relaxed case $(\mu=1)$. Regardless of the speed of convergence, the optimal parameters are found for all values of $0<\mu \leqslant 1$ (Fig. 4). When $\sigma_{b} \neq 0$, the algorithm estimates the system parameters more precisely if $\mu$ is small. In other words, when $\mu$ increases the algorithm speed increases, however the precision of the estimation decreases. Therefore, in the presence of measurement noise, it is necessary to make a good compromise between the rate of convergence and the desired estimation quality. Using an adaptive relaxation coefficient (for example, a decreasing sequence as in classical LMS approaches) may also yield good results. Note that the starting point of the algorithm $\hat{\boldsymbol{\theta}}_{1}=$ $\left[\begin{array}{llll}0 & 0 & 0 & 1\end{array}\right]$ does not verify assumption 1 (i.e. $v_{1}<0$ ). Nevertheless, the convergence takes place (Fig. 4). Similar results are also obtained for different initial points, whether they verify assumption 1 or not, and regardless of the value of $\mu$ and $\sigma_{b}$.

Now, assumption 2 is put to the test. The second part of this assumption (the $\alpha$-mixing condition) is easy to obtain in practice by using an input sequence having a finite correlation length. On the other hand, if the input signal is quantized (on a finite number of bits, $n$ ), the first condition of assumption 2 cannot be verified, since there only exists $2^{n L}$ different $\phi_{k}$. Note that the quan- 


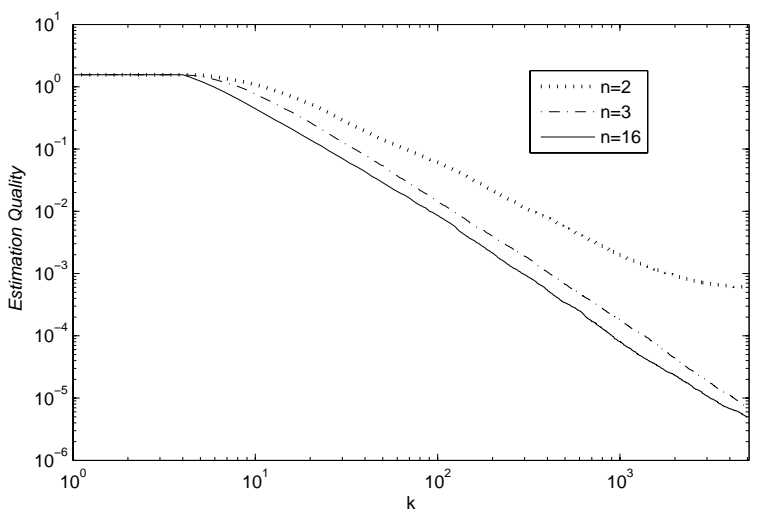

Fig. 5. $80^{t h}$ percentile of $\left(1-v_{k}\right)$ for three values of $n$ with $\mu=1$ and $\sigma_{b}=0$.

tization levels of the input signal should not include zero, because the probability of having $\left\|\phi_{k}\right\|=0$, which causes $\alpha_{k}$ to be undefined, would then be non-zero. This problem can also be tackled by adding a small positive constant in the denominator of $\alpha_{k}$ as in the normalized LMS algorithm (Haykin, 2001).

Figure 5 shows the estimation quality, defined as above, for $n=2,3$ and 16 bits, in the absence of measurement noise. As expected, the larger $n$ is, the finer the estimation is. In this case, $n=3$ yields very satisfactory results, nonetheless. Finally, it should be noted that, when $L$ is large, a very coarse quantization of the input signal can still yield some very good results (the number of possible $\phi_{k}$ increases exponentially with $L$, whereas the number of parameters to be estimated increases linearly). A result of similar nature was established and verified in (Juillard \& Colinet, 2007).

\section{Conclusion}

In this paper, we introduced LIMBO, a recursive parameter estimation method using binary observations based on the LMS algorithm. The asymptotical convergence of the method was investigated. In particular, we gave some assumptions under which the convergence of the algorithm to the optimal system parameters is theoretically guaranteed. We showed by simulation that these assumptions do not necessarily have to be met in practice in order to obtain a good performance of the method. This method has a very low computational complexity and implementation cost, making it appropriate for use in the context of micro-electronics. The extension of this approach to more complex systems (IIR systems), the study of the convergence rate and the design of an optimal input signal are the subject of future works.

\section{Acknowledgements}

This research was partly funded by the ARFU program of the French National Research Agency (ANR), as a part of the SYSRECAP project.

\section{References}

Bai, E.-W. \& Reyland Jr., J. (2008). Towards identification of wiener systems with the least amount of a priori information on the nonlinearity. Automatica, 44(4), 910-919.

Colinet, E. \& Juillard, J. (2010). A weighted leastsquares approach to parameter estimation problems based on binary measurements. IEEE Transactions on Automatic Control, 55(1), 148-152.

Deb, N. (2005). Defect Oriented Test of Inertial Microsystems. PhD thesis, Carnegie Mellon University.

Gustafsson, F. \& Karlsson, R. (2009). Statistical results for system identification based on quantized observations. Automatica, 45(12), 2794-2801.

Haykin, S. (2001). Adaptive Filter Theory. NY: PrenticeHall.

Jafari, K., Juillard, J., \& Colinet, E. (2010). A recursive system identification method based on binary measurements. Proceedings of the 49th IEEE Conference on Decision and Control, (pp. 1154-1158).

Juillard, J. \& Colinet, E. (2007). Initialization of the BIMBO self-test method using binary inputs and outputs. Proceedings of the 46 th IEEE Conference on Decision and Control, (pp. 578-583).

Juillard, J., Jafari, K., \& Colinet, E. (2009). Asymptotic consistency of weighted least-square estimators for parameter estimation problems based on binary measurements. Proceedings of the 15th IFAC Symposium on System Identification, (pp. 72-77).

Ljung, L. (1999). System identification - theory for the user. Upper Saddle River: Prentice Hall.

Rafajlowicz, E. (1996). Linear systems identification from random threshold binary data. IEEE Transactions on Signal Processing, 44(8), 2064-2070.

Rosenblatt, M. (1956). A central limit theorem and a strong mixing condition. Proceedings of the National Academy of Sciences of the USA, 42, 43-47.

Van de Plassche, R. (2005). CMOS integrated analog-todigital and digital-to-analog converters. NY: SpringerVerlag.

Walter, E. \& Pronzato, L. (1997). Identification of parametric models from experimental data. Springer.

Wang, L., Yin, G., \& Zhang, J. (2006). Joint identification of plant rational models and noise distribution functions using binary-valued observations. Automatica, 42(4), 535-547.

Wang, L., Zhang, J., \& Yin, G. (2003). System identification using binary sensors. IEEE Transactions on Automatic Control, 48(11), 1892-1907.

Wigren, T. (1997). ODE analysis and redesign in blind adaptation. IEEE Transactions on Automatic Control, 42(12), 1742-1747.

Wigren, T. (1998). Adaptive filtering using quantized output measurements. IEEE Transactions on Signal Processing, 46(12), 3423-3426.

Zhao, Y., Wang, L., Yin, G., \& Zhang, J. (2007). Identification of wiener systems with binary-valued output observations. Automatica, 43(10), 1752-1765. 